\title{
Transcriptome analysis reveals the host response to Schmallenberg virus in bovine cells and antagonistic effects of the NSs protein
}

\author{
Anne-Lie Blomström ${ }^{1,2 *}$, Quan Gu², Gerald Barry ${ }^{2,3}$, Gavin Wilkie², Jessica K Skelton², Margaret Baird²,
} Melanie McFarlane², Esther Schnettler ${ }^{2}$, Richard M Elliott ${ }^{2}$, Massimo Palmarini ${ }^{2}$ and Alain Kohl²

\begin{abstract}
Background: Schmallenberg virus (SBV) is a member of the Orthobunyavirus genus (Bunyaviridae family) causing malformations and abortions in ruminants. Although, as for other members of this family/genus, the non-structural protein NSs has been shown to be an interferon antagonist, very little is known regarding the overall inhibitory effects and targets of orthobunyavirus NSs proteins on host gene expression during infection. Therefore, using RNA-seq this study describes changes to the transcriptome of primary bovine cells following infection with Schmallenberg virus (SBV) or with a mutant lacking the non-structural protein NSs (SBVdeINSs) providing a detailed comparison of the effect of NSs expression on the host cell.

Results: The sequence reads from all samples (uninfected cells, SBV and SBVdeINSs) assembled well to the bovine host reference genome (on average 87.43\% of the reads). During infection with SBVdelNSs, 649 genes were differentially expressed compared to uninfected cells (78.7\% upregulated) and many of these were known antiviral and IFN-stimulated genes. On the other hand, only nine genes were differentially expressed in SBV infected cells compared to uninfected control cells, demonstrating the strong inhibitory effect of NSs on cellular gene expression. However, the majority of the genes that were expressed during SBV infection are involved in restriction of viral replication and spread indicating that SBV does not completely manage to shutdown the host antiviral response.

Conclusions: In this study we show the effects of SBV NSs on the transcriptome of infected cells as well as the cellular response to wild type SBV. Although NSs is very efficient in shutting down genes of the host innate response, a number of possible antiviral factors were identified. Thus the data from this study can serve as a base for more detailed mechanistic studies of SBV and other orthobunyaviruses.
\end{abstract}

Keywords: Schmallenberg virus, Orthobunyavirus, RNA-seq, Transcriptome, Antiviral response, Non-structural protein NSs

\section{Background}

Schmallenberg virus (SBV) is a negative-sense, singlestranded, segmented, RNA virus belonging to the Bunyaviridae family, within the genus Orthobunyavirus. The virus was discovered 2011 in connection with outbreaks of diarrhoea, reduced milk production and fever in cattle

\footnotetext{
* Correspondence: anne-lie.blomstrom@slu.se

'Section of Virology, Department of Biomedical Sciences and Veterinary Public Health, Swedish University of Agricultural Sciences, 75007 Uppsala, Sweden

${ }^{2}$ MRC-University of Glasgow Centre for Virus Research, Glasgow G61 1QH, Scotland, UK

Full list of author information is available at the end of the article
}

in Germany and the Netherlands [1] and has since the initial discovery rapidly spread to many European countries [2]. Although infection is often connected with milder symptoms it can cause foetal abortions and malformations in ruminants [1,3]. SBV was the first orthobunyavirus detected in Europe, but the genus consists of over $170 \mathrm{vi}-$ ruses grouped into 18 serogroups and many of these are important pathogens. In humans, this includes for example La Crosse virus (LACV) causing encephalitis and Ngari Virus causing haermorrhagic fever [4]. Animal pathogens, apart from SBV, includes Akabane virus (AKBV)

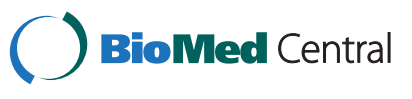


and Cache Valley virus (CVV) that also infect ruminants and can cause abortions and congenital malformations.

The SBV genome consists of three segments (L, large; M, medium; and S, small) coding for four structural proteins (N, nucleoprotein; Gn and Gc, glycoproteins; L, RNAdependent RNA polymerase) and two non-structural proteins (NSm and NSs). Through the production of recombinant viruses lacking NSs (LACVdelNSs, AKBVdelNSs and Bunyamwera(BUNV)delNSs), NSs has been shown to be a major virulence factor for orthobunyaviruses as these deletant viruses are attenuated and show reduced growth rate compared to wild type virus [5-7]. SBV NSs has also been shown to be able to counteract host antiviral responses. Recombinant SBV lacking ability to express NSs (SBVdelNSs) is a potent inducer of type I IFN while SBV does not induce the IFN response after infection $[8,9]$. Apart from interfering with the IFN pathway, SBV NSs has also been shown to induce degradation of the RPB1 subunit of RNA polymerase II and to potentially have a pro-apoptotic role [10]. However, little is known about the overall inhibitory effects and targets of orthobunyavirus NSs proteins on gene expression in infected cells. Therefore, in this study we analysed the changes in the transcriptome of primary bovine cells following infection with either wild type SBV or SBVdelNSs $(5,6)$.

\section{Results and discussion}

Viral infection and sequencing output - quality and reference assembly

We carried out RNA-seq analysis in infected and mockinfected primary bovine cells, in order to use cells with an intact cell-autonomous innate immune system. Bovine primary fibroblasts were infected with either SBV, SBVdelNSs or mock-infected and RNA was extracted at $16 \mathrm{~h}$ p.i.. Three biological replicates were assesses for each sample and cells were also infected in parallel for immunofluorescence (Figure 1A) and Western blot (Figure 1B) in order to confirm that the bovine primary cells were infected by SBV and SBVdelNSs at $16 \mathrm{~h}$. Both the SBV and the SBVdelNSs infected cells were positive for the virus with an estimated infection rate at about $30 \%$.

The samples for transcriptome analysis were sequenced on Illumina MiSeq. On average, 26.5 million reads/sample were generated with a phred quality value of 30 or higher and $87.43 \%$ of the sequence reads assembled against the Bos taurus genome (Ensembl Btau_4.0) using TopHat2 [11]. mRNA enrichment was carried out prior

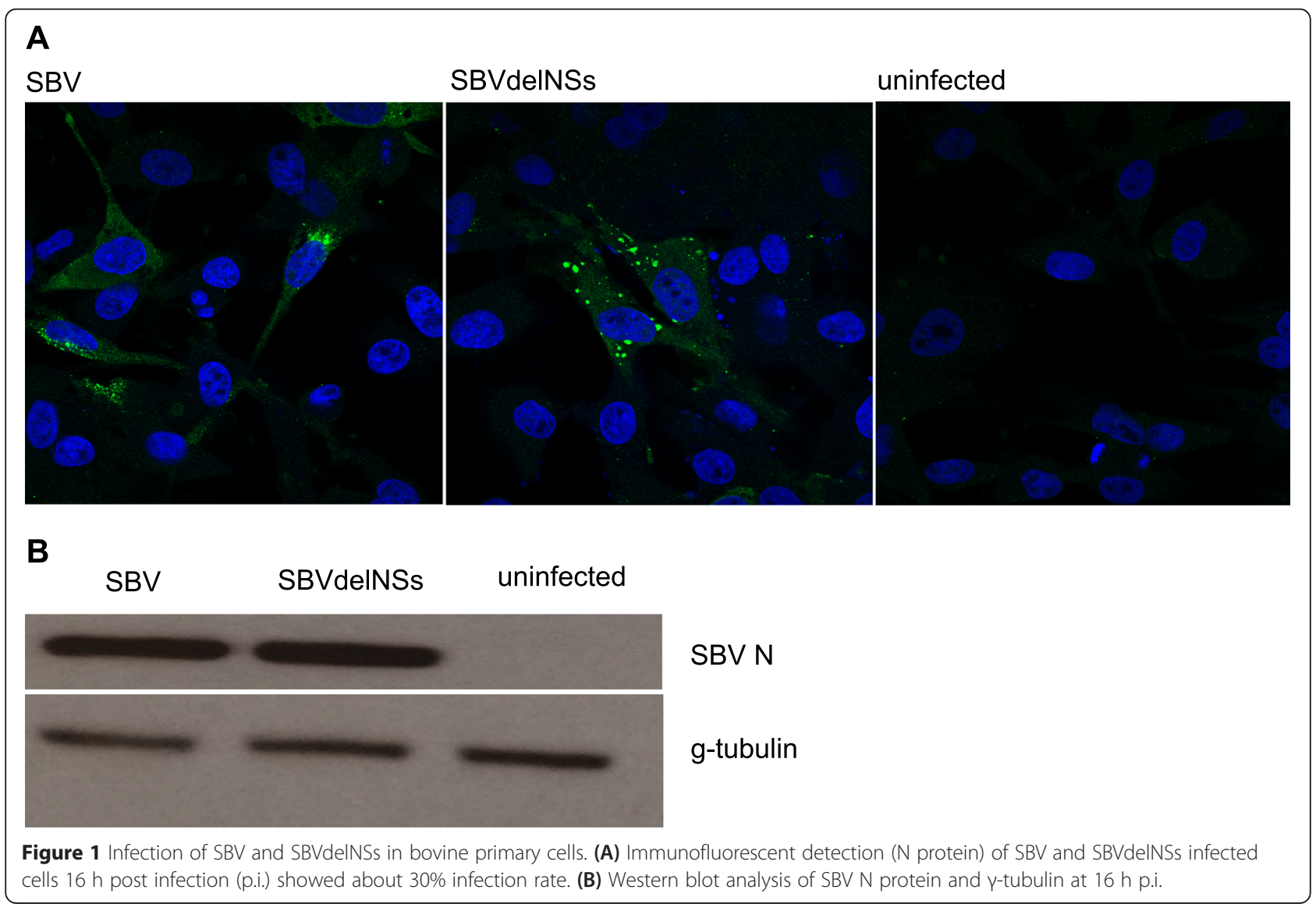


to sequencing and bunyaviruses in general (including SBV) lack a poly-A tail in their genome and mRNAs, consequently no assembly against the virus genome was performed.

\section{Differential expression analysis}

Cuffdiff2 [12] was used to identify differentially expressed (DE) genes (genes with a $+/-2$-fold change or more and with $\mathrm{p} \leq 0.05$ were considered significant). $651 \mathrm{DE}$ genes were identified and most were found in the SBVdelNSs infected cells (Figure 2A). Hence, the majority of the DE genes are as a direct or indirect result of the loss of NSs. Most (78.7\%) of the DE genes affected by the loss of NSs were upregulated and fold differences ranged from 12.7fold to the 1-fold cut-off value (on a $\log 2$ scale). The fold changes for down-regulated genes $(21.3 \%)$ were more subtle, ranging from 2.72 -fold to the 1 -fold cut-off $(\log 2$ scale). The DE sequence analysis was validated for 10 genes using Sybr green realtime PCR (Figure 2B); the fold changes for SBVdelNSs compared to the SBV infected cells were all significant $(\mathrm{p} \leq 0.05)$ and corresponded to the sequencing data.

\section{Pathway analysis}

Ingenuity Pathway Analysis (IPA) (http://www.ingenuity. com/products/ipa) showed that the DE genes are, to a great extent, involved in pathways associated with host antiviral responses (Table 1), such as type I IFN-signalling and IFN-dependent gene expression, as well as pattern recognition. This is also evident when extracting the top 30 most upregulated genes (Table 2) as most of these have antiviral functions. The major molecules involved in viral
RNA recognition including DDX58 (RIG-I), TRIM25, IFIH1 (MDA5), PKR, TLR3 were highly up-regulated in SBVdelNSs infected cells (Figure 3A), as are those involved in antigen presentation to CD8+ T-lymphocytes including MHC I $\alpha / \beta$ and TAP $1 / 2$ (Figure 3B). As a consequence of activation of the viral RNA recognition pathways many interferon stimulated genes such OAS1/2, MX1 and several guanylate binding proteins, were upregulated [13-16]. Several interleukins were found among the DE transcripts, for example IL-8 and its downstream molecules (e.g. VCAM-1, ICAM-1 and Cox2) involved in inflammation, activation of angiogenesis and leucocyte infiltration/activation (Figure 3B).

In SBV-infected cells only nine DE genes (RSAD2, ISG15, OAS1, OAS2, IFIT2, MX1, GNAL, RPS3A and MDFI) were identified (eight upregulated and one downregulated) (Figure $2 \mathrm{~A}$ and Table 1 ). The majority of these genes are involved in antiviral responses. All up-regulated genes in SBV infected cells, except guanine nucleotide binding protein (G protein), alpha activating activity polypeptide, olfactory type (GNAL) and ribosomal protein S3A (RPS3A), were also upregulated following SBVdelNSs infection, although to a much higher extent, indicating that NSs may not be able to completely shutdown all genes of the host antiviral response. Viperin (RSAD2) can be induced by a number of different viruses such as sindbis virus, Japanese encephalitis virus and lassa fever virus (LASV) either dependently and independently of IFN [17]. For example, Zapata JC et al. [18] showed that LASV strongly induce viperin early in infection (PBMC) while the attenuated ML29 has a weaker and delayed viperin induction suggesting that ML29 has a mechanism to

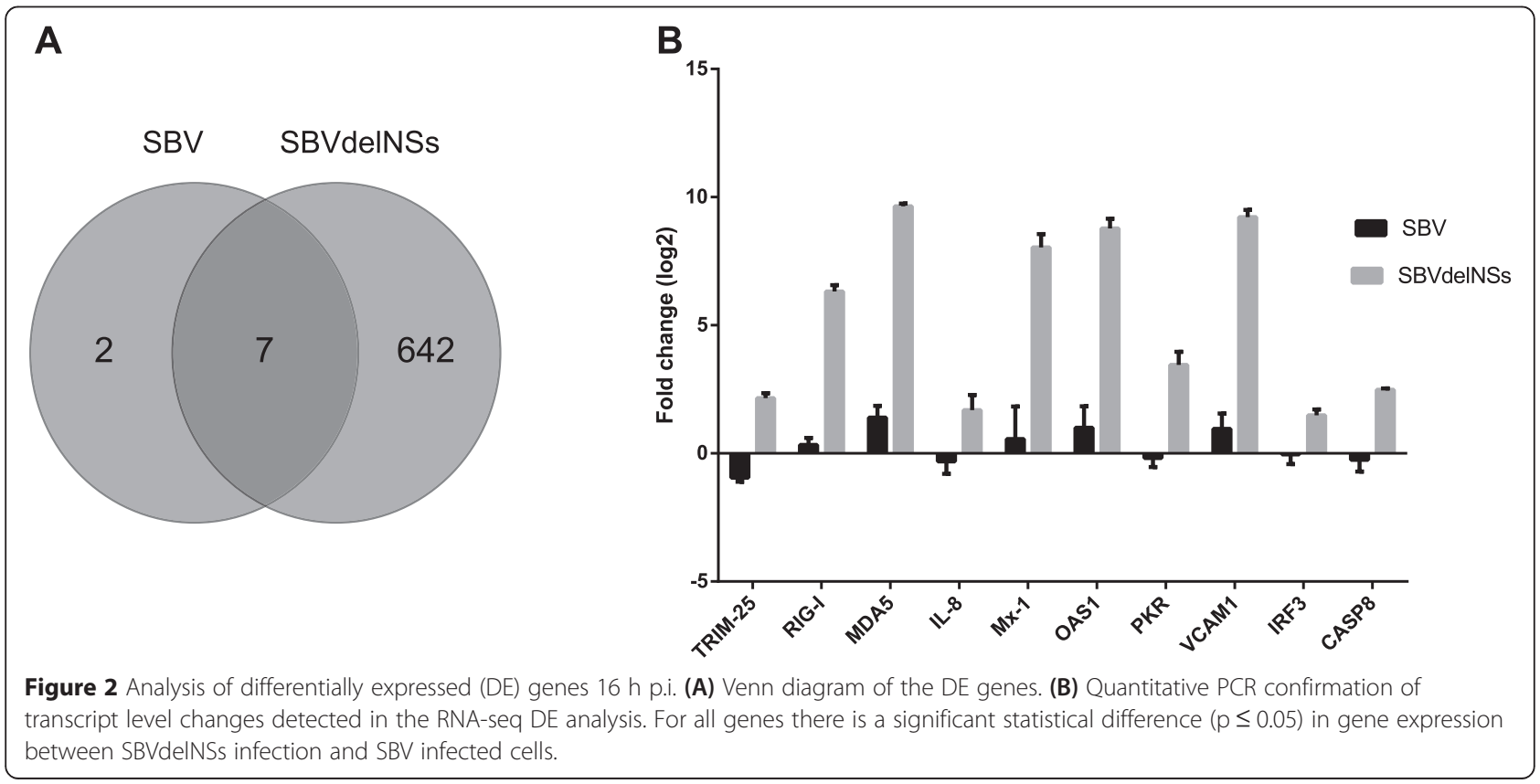


Table 1 Canonical pathway associated with infection of Schmallenberg virus or a mutant lacking NSs

\begin{tabular}{|c|c|c|c|}
\hline \multicolumn{4}{|l|}{ SBV vs uninfected } \\
\hline Ingenuity canonical pathways & -log(p-value) & Ratio & Genes \\
\hline Interferon Signaling & 3.96E00 & $5.56 \mathrm{E}-02$ & OAS1,MX1 \\
\hline $\begin{array}{l}\text { Activation of IRF by Cytosolic Pattern } \\
\text { Recognition Receptors }\end{array}$ & $3.42 \mathrm{E} 00$ & $2.74 \mathrm{E}-02$ & IFIT2,ISG15 \\
\hline $\begin{array}{l}\text { Role of Pattern Recognition Receptors in } \\
\text { Recognition of Bacteria and Viruses }\end{array}$ & 3.06E00 & $1.83 \mathrm{E}-02$ & OAS1,OAS2 \\
\hline $\begin{array}{l}\text { Role of Lipids/Lipid Rafts in the Pathogenesis } \\
\text { of Influenza }\end{array}$ & $1.95 \mathrm{E} 00$ & 3.45E-02 & RSAD2 \\
\hline Ephrin B Signaling & 1.47E00 & $1.22 \mathrm{E}-02$ & GNAL \\
\hline CDK5 Signaling & 1.39E00 & $1.03 \mathrm{E}-02$ & GNAL \\
\hline G Beta Gamma Signaling & 1.39E00 & $8.26 \mathrm{E}-03$ & GNAL \\
\hline IL-1 Signaling & 1.37E00 & $9.17 \mathrm{E}-03$ & GNAL \\
\hline Androgen Signaling & 1.29E00 & $6.9 \mathrm{E}-03$ & GNAL \\
\hline Relaxin Signaling & $1.2 \mathrm{E} 00$ & $6.1 \mathrm{E}-03$ & GNAL \\
\hline \multicolumn{4}{|l|}{ SBVdelNSs vs uninfected } \\
\hline Ingenuity Canonical Pathways & -log(p-value) & Ratio & Genes \\
\hline Interferon Signaling & 9.77E00 & 3.33E-01 & $\begin{array}{l}\text { IFIT3,SOCS1,IFIT1,OAS1,MX1,IFI35,STAT2,IRF9,PSMB8,JAK2, } \\
\text { TAP1,IRF1 }\end{array}$ \\
\hline $\begin{array}{l}\text { Activation of IRF by Cytosolic Pattern } \\
\text { Recognition Receptors }\end{array}$ & 9.32E00 & $2.05 \mathrm{E}-01$ & $\begin{array}{l}\text { ZBP1,IRF9,TBK1,IL6,IRF3,ADAR,NFKB1,ISG15,IFIH1,NFKBIA, } \\
\text { CD40,DDX58,STAT2,IFIT2,NFKBIB }\end{array}$ \\
\hline $\begin{array}{l}\text { Role of Pattern Recognition Receptors in } \\
\text { Recognition of Bacteria and Viruses }\end{array}$ & 8.35E00 & $1.56 \mathrm{E}-01$ & $\begin{array}{l}\text { OAS1,PRKCQ,C3,OAS2,IL6,CCL5,IRF3,NFKB1,RNASEL,TLR2, } \\
\text { IFIH1,TLR4,DDX58,EIF2AK2,TLR3,RIPK2,ATM }\end{array}$ \\
\hline NF-kB Signaling & 7.81E00 & $1.22 \mathrm{E}-01$ & $\begin{array}{l}\text { AZ12,PRKCQ,RELB,IL36A,TNFAIP3,TBK1,IRAK3,NFKB1,TLR2, } \\
\text { TLR4,TNIP1,NFKBIA,CD40,NGFR,MAP3K8,TRAF5,EIF2AK2, } \\
\text { TLR3,NFKBIB,CASP8,ATM,TNFRSF11B }\end{array}$ \\
\hline $\begin{array}{l}\text { Role of Macrophages, Fibroblasts and } \\
\text { Endothelial Cells in Rheumatoid Arthritis }\end{array}$ & 7.74E00 & 8.77E-02 & $\begin{array}{l}\text { SOCS1,ICAM1,IL1RL1,IL6,CCL5,JAK2,NFKB1,FCGR1A, } \\
\text { IL18R1,Prss2,NFKBIA,CCL2,NGFR,TLR3,TRAF5,NFKBIB,ATM, } \\
\text { TNFRSF1 1B,IL8,VCAM1,PRKCQ,WNT2B,IL15,IL36A,IRAK3, } \\
\text { IL7,TLR2,IL16,,LR4,CSF1 }\end{array}$ \\
\hline Dendritic Cell Maturation & 7.58E00 & $1.04 \mathrm{E}-01$ & $\begin{array}{l}\text { B2M,ICAM1,HLA-A,RELB,IL15,IL36A,HLA-DMB,IL6,JAK2, } \\
\text { NFKB1,FCGR1A,TLR2,TLR4,NFKBIA,CD40,NGFR,STAT2, } \\
\text { TLR3,NFKBIB,IL23A,ATM,TNFRSF11B }\end{array}$ \\
\hline Antigen Presentation Pathway & $6.97 \mathrm{E} 00$ & $2.38 \mathrm{E}-01$ & $\begin{array}{l}\text { B2M,PSMB9,NLRC5,HLA-A,HLA-DMB,PSMB8,TAP1,TAP2, } \\
\text { TAPBP,MR1 }\end{array}$ \\
\hline $\begin{array}{l}\text { Role of PKR in Interferon Induction and } \\
\text { Antiviral Response }\end{array}$ & $6.62 \mathrm{E} 00$ & $2.04 \mathrm{E}-01$ & $\begin{array}{l}\text { NFKBIA,TRAF5,EIF2AK2,TLR3,CASP8,NFKBIB,NFKB1, } \\
\text { RNASEL,FCGR1A,IRF1 }\end{array}$ \\
\hline LXR/RXR Activation & $6.08 \mathrm{E} 00$ & 1.15E-01 & $\begin{array}{l}\text { C3,IL1RL1,IL36A,IRF3,IL6,NFKB1,ABCA1,TLR4,CCL2,NGFR, } \\
\text { MYLIP,LPL,CD14,PTGS2,TLR3,TNFRSF11B }\end{array}$ \\
\hline Hepatic Fibrosis/Hepatic Stellate Cell Activation & 5.93E00 & $1.1 \mathrm{E}-01$ & $\begin{array}{l}\text { IL8,VCAM1,ICAM1,IL1RL1,IGFBP5,IL6,CCL5,NFKB1,TLR4, } \\
\text { CD40,CCL2,CSF1,HGF,NGFR,TGFB2,CD14,TNFRSF1 1B }\end{array}$ \\
\hline
\end{tabular}

This table shows the top 10 IPA canonical pathways associated with SBV and SBVdeINSs infection, the p-value of Fisher's exact test, the ratio (number of the genes in the dataset mapping to each specific pathway divided by the total number of genes in the pathway) and lists the DE genes from this study involved in respective pathway.

interfere with the host-response signalling pathways. Antiviral activity of RSAD2 has been shown against for example BUNV, chikungunya virus, influenza virus and dengue virus through different mechanisms such as inhibition of viral replication and budding/egress [13-16,19]. MX1 has also been shown to have antiviral activity against a number of different RNA viruses such influenza and bunyaviruses [20]. MX1 is significantly upregulated in foetuses infected with CVV and it has been proposed to be involved in the clearance of the virus [21]. During LACV infection MX1 can bind to the nucleocapsid and inhibit viral replication [22]. Also, ISG15 is upregulated during foetal infection with CVV [21]. The OAS proteins performs their antiviral activity through the activation of RNAseL which leads to degradation of cellular and viral RNA [23] and OAS1, but not OAS2, is reported to contribute to a slight inhibition of BUNV and BUNVdelNSs viruses [19]. Hence although very few genes were up 
Table 2 Up-regulated genes in SBV or SBVdeINSs infected cells

\begin{tabular}{|c|c|c|c|}
\hline \multicolumn{4}{|c|}{ Top up-regulated genes } \\
\hline \multirow[t]{2}{*}{ Symbol } & \multirow[t]{2}{*}{ Entrez gene name } & \multicolumn{2}{|c|}{ Fold change (log2) } \\
\hline & & SBV & delNSsSBV \\
\hline RSAD2 & radical S-adenosyl methionine domain containing 2 & 1.96 & 11.518 \\
\hline ISG15 & ISG15 ubiquitin-like modifier & 1.86 & 10.886 \\
\hline $\mathrm{IFIH1/MDA5}$ & interferon induced with helicase $C$ domain 1 & - & 9.987 \\
\hline GBP5 & guanylate binding protein 5 & - & 9.837 \\
\hline OAS1 & 2'-5'-oligoadenylate synthetase $1,40 / 46 \mathrm{kDa}$ & 2.24 & 9.818 \\
\hline IFIT2 & interferon-induced protein with tetratricopeptide repeats 2 & 1.06 & 9.643 \\
\hline $\mathrm{IFI} 27$ & interferon, alpha-inducible protein 27 & - & 9.387 \\
\hline ZBP1 & Z-DNA binding protein 1 & - & 9.372 \\
\hline GBP4 & guanylate binding protein 4 & - & 9.354 \\
\hline VCAM1 & vascular cell adhesion molecule 1 & - & 9.287 \\
\hline OAS2 & 2'-5'-oligoadenylate synthetase 2,69/71 kDa & 2.41 & 9,064 \\
\hline IFIT3 & interferon-induced protein with tetratricopeptide repeats 3 & - & 8.87 \\
\hline$M \times 1$ & myxovirus (influenza virus) resistance 1, & 1.45 & 8.683 \\
\hline XAF1 & XIAP associated factor 1 & - & 8.264 \\
\hline CCL5 & chemokine ( $\mathrm{C}-\mathrm{C}$ motif) ligand 5 & - & 8.081 \\
\hline RTP4 & receptor (chemosensory) transporter protein 4 & - & 8.059 \\
\hline GBP1 & guanylate binding protein 1, interferon-inducible & - & 7.483 \\
\hline GBP2 & guanylate binding protein 2 , interferon-inducible & - & 6.853 \\
\hline LGALS9 & lectin, galactoside-binding, soluble, 9 & - & 6.571 \\
\hline USP18 & ubiquitin specific peptidase 18 & - & 6.536 \\
\hline CMPK2 & cytidine monophosphate (UMP-CMP) kinase 2, mitochondrial & - & 6.52 \\
\hline UBA7 & ubiquitin-like modifier activating enzyme 7 & - & 6.15 \\
\hline DDX58 & DEAD (Asp-Glu-Ala-Asp) box polypeptide 58 & - & 6.022 \\
\hline CXCL2 & chemokine (C-X-C motif) ligand 2 & - & 5.946 \\
\hline CYP2J2 & cytochrome P450, family 2, subfamily J, polypeptide 2 & - & 5.936 \\
\hline IFl6 & interferon, alpha-inducible protein 6 & - & 5.807 \\
\hline MCHR1 & melanin-concentrating hormone receptor 1 & - & 5.762 \\
\hline IFIT1 & interferon-induced protein with tetratricopeptide repeats 1 & - & 5.681 \\
\hline HERC6 & HECT and RLD domain containing E3 ubiquitin protein ligase family member 6 & - & 5.612 \\
\hline BATF2 & basic leucine zipper transcription factor, ATF-like 2 & - & 5.483 \\
\hline GNAL & Guanine nucleotide-binding protein G(olf) subunit alpha & 1.37 & - \\
\hline RPS3A & ribosomal protein $\mathrm{S} 3 \mathrm{~A}$ & 1.16 & - \\
\hline
\end{tabular}

The table indicates the most up-regulated genes during SBV and SBVdelNSs infection of bovine primary cells at $16 \mathrm{~h}$ (compared to uninfected cells). The foldchange is shown in log 2 and "-" denotes no fold-change.

regulated during SBV infection most of the upregulated genes have all been previously shown to have antiviral activity for other similar viruses indicating that these genes, individually or collectively, may have an important role in the antiviral response and the consequent restriction of SBV infection. However, it should be noted that we only investigated host responses at one time point, and it is possible that at an earlier or later time point during the viral replication cycle, more DE genes may be found, as shown for dengue virus for example [24,25]. As not all primary cells were infected by SBV or SBVdelNSs it is possible that weak host responses to SBV might be masked by transcripts from uninfected cells. This may also explain why the DE analysis of SBV infected cells did not show a general host transcription shutdown as described [10], although mRNA turnover and cell types used in this and other studies may be further reasons. Only one transcript (MDFI - MyoD family inhibitor) was downregulated in SBV infected cells compared to uninfected cells. This gene was also down-regulated in SBVdelNSs- 


\section{A}

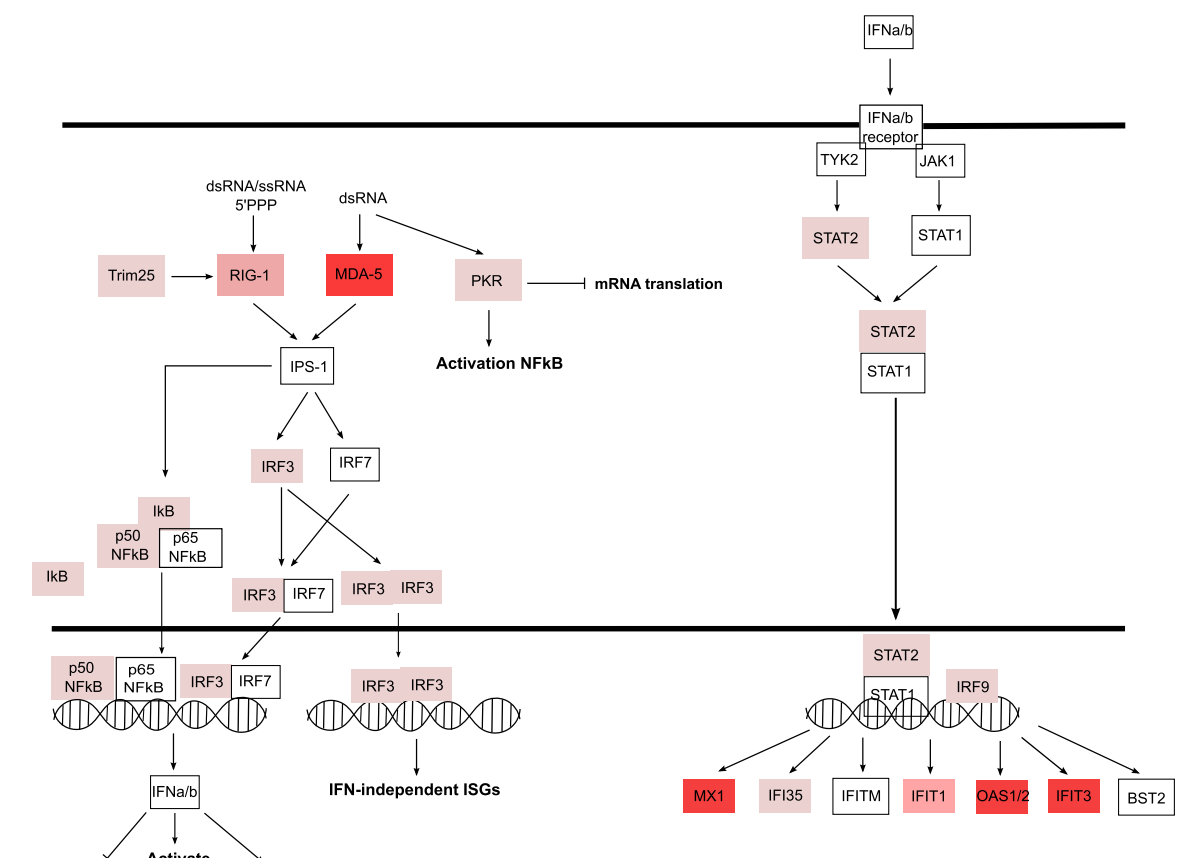

Viral replication immune cells Antigen presentation

Antiviral effectors, negative and positive regulators of IFN signaling

B

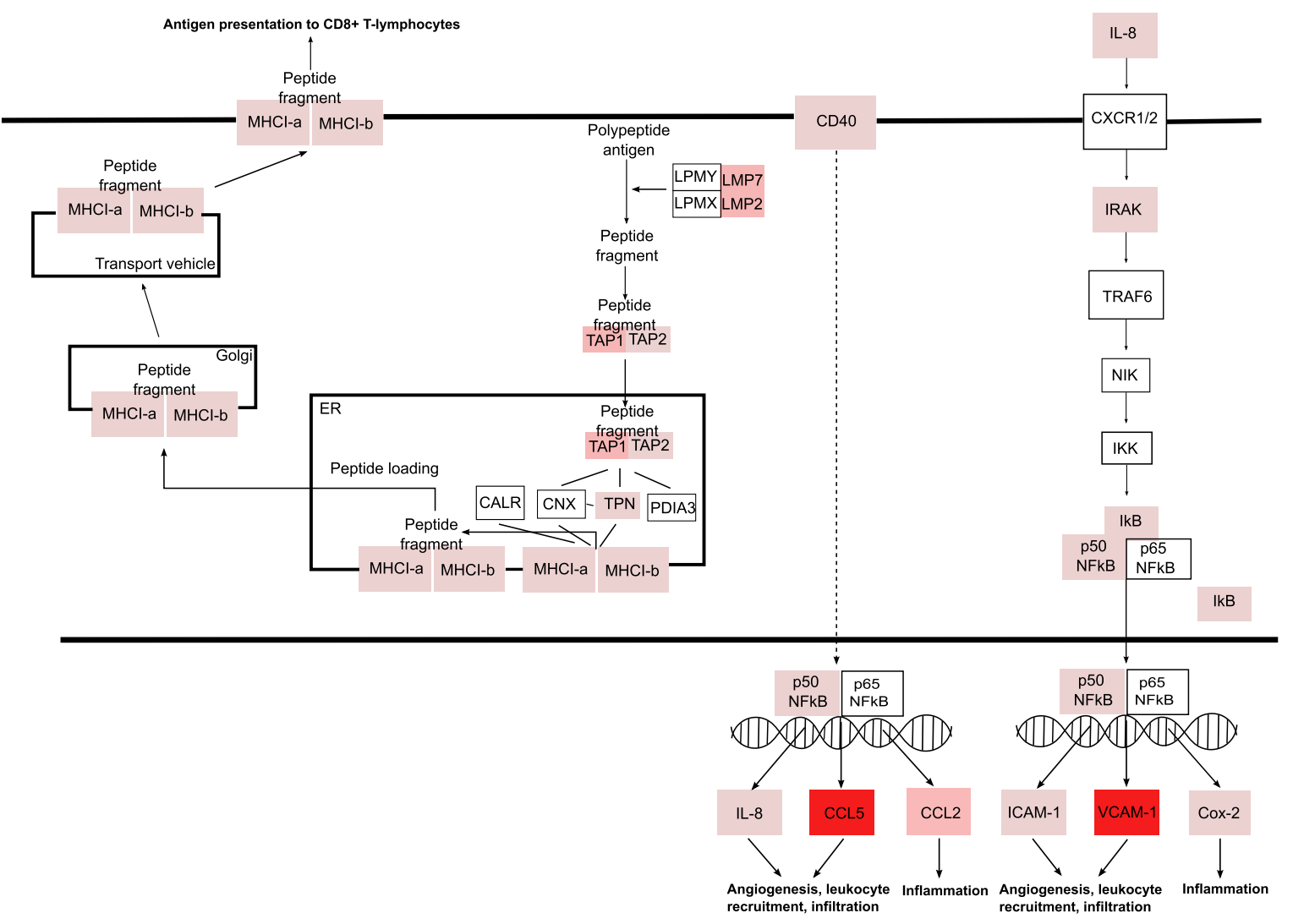

Figure 3 Schematic overview of some of the most significant IPA-identified, canonical host response pathways. (A) Role of pattern recognition pathways and the interferon signaling pathway. (B) Antigen presentation pathways and pathways involved in leukocyte recruitment and IL-8 signaling. Red boxes are genes upregulated in SBVdelNSs compared to uninfected cells; white boxes are genes not differentially expressed. 
infected cells, indicating that this change is not due to the NSs protein alone. This is a transcription factor that negatively regulates myogenic proteins. Knockout studies of the murine homolog, inhibitory of myogenic family (l-mfa), have shown that lack of l-mfa can lead to embryonic lethality and placental defects as well as skeletal patterning defects [26]. Although, the high viral levels of SBV seen in brain tissue are likely to cause the teratogenic effects seen during SBV infection the downregulation of MDFI in SBV and SBVdelNSs infected cells is potentially of interest in relation to foetal abnormalities linked to SBV infection. Future studies will determine whether SBV and SBVdelINSs affect expression of MDFI in fetal brain tissue.

\section{Conclusions}

We have investigated the effect of SBV infection on the host transcriptome and more specifically the effects induced by lack of NSs expression. Our results show the response to orthobunyavirus infection in a relevant host cell system and that NSs is very efficient in shutting down the immune response of the host but that despite this a number of known antiviral proteins (such as viperin, MX1, OAS1/2 etc.) are still induced during SBV infection. The data from this study serves to identify possible antiviral factors and serve as a basis for more detailed mechanistic studies of SBV and other orthobunyaviruses as well as the mechanism(s) of action of their NSs protein.

\section{Methods \\ Cells}

Bovine fibroblast cells were isolated from cow aortas by collagenase treatment using a method adapted described earlier [27]. Aortas were harvested from killed animals sourced from an abattoir and incubated at room temperature for $3 \mathrm{~h}$ in Dulbecco's modified Eagles medium (DMEM) (Life Technologies) supplemented with $5 \%$ foetal bovine serum (FBS), $100 \mathrm{U} / \mathrm{ml}$ penicillin $100 \mu \mathrm{g} / \mathrm{ml}$ streptomycin (P/S) and $2.5 \mu \mathrm{g} / \mathrm{ml}$ amphotericin B. After incubation, the aortas were cleaned, opened longitudinally and placed, intima layer down, into collagenase $(2 \mathrm{mg} / \mathrm{ml}$, DMEM) for $60 \mathrm{~min}$ at $37^{\circ} \mathrm{C}$. After incubation the cells were isolated by collection of the collagenase supernatant and scraping of the aorta wall that was in contact with the collagenase. The supernatant containing the cells was centrifuged at $1000 \mathrm{rpm}$ for 5 mins. The cells were then resuspended, seeded in 12-well plates and maintained at $37^{\circ} \mathrm{C}$ and $5 \%$ $\mathrm{CO} 2$ in DMEM with $20 \% \mathrm{FBS}, 100 \mathrm{U} / \mathrm{ml}$ penicillin $100 \mu \mathrm{g} / \mathrm{ml}$ Streptomycin, $2.5 \mu \mathrm{g} / \mathrm{ml}$ amphotericin B. The population was initially a mixture of endothelial cells and fibroblasts but frequent passaging for approximately 10 days allowed the fibroblasts to outgrow the endothelial cells and form a pure population of fibroblasts.

\section{Viruses and infections}

Rescued wild type SBV as well as a SBV lacking NSs described in Elliot et al. [8] were used throughout all the experiments.

Bovine fibroblasts were seeded in 24-well plates and at a confluence of $80 \%$ the cells were infected with SBV or SBVdelNSs at a MOI of 5 for $16 \mathrm{~h}$. Mock-infected cells were included in parallel as control. For each condition three individual replicates were set up. Viral infection was confirmed by immunofluorescence and western blot targeting the $\mathrm{N}$-protein of the virus. For western blot $\gamma$ tubulin (Sigma) was used as an internal control.

\section{RNA extraction}

RNA was extracted from infected and uninfected cells using a combination of Trizol (Life Technology) and RNeasy Mini kit (Qiagen). In short, the cell media was discarded from the wells and the cells were mixed and homogenised in $750 \mu \mathrm{l}$ Trizol. Chloroform $(150 \mu \mathrm{l})$ was added and the sample mixed prior to a short incubation at room temperature and a centrifugation step at $+4^{\circ} \mathrm{C}$ for 15 minutes. The upper aqueous phase was transferred to a new tube and mixed with $70 \% \mathrm{EtOH}$ and then transferred to a RNeasy Mini Spin Column. The sample was bound to the column through centrifugation and then washed once with RW1 buffer and twice with RPE-buffer before eluted with $30 \mu \mathrm{l}$ RNase free water. The RNA was stored at $-80^{\circ} \mathrm{C}$ until further use.

\section{Library preparation and MiSeq sequencing}

The TruSeq stranded mRNA sample preparation kit (Illumina) was used to enrich samples for mRNA and construct libraries for sequencing. Single end datasets with a read length of 150 nucleotides were generated from each sample library on an Illumina MiSeq. Each sample (SBV, SBVdelNSs and uninfected control) was set up in triplicate, and sequenced on a separate MiSeq run, with 9 runs being carried out in total. The sequences from the MiSeq runs have been deposited in the European Nucleotide Archive (ENA) and can be accessed through the study accession number PRJEB9007.

\section{Sequence quality and assembly}

The FastQC software (http://www.bioinformatics.babra ham.ac.uk/projects/fastqc) was used to check the RNASeq reads quality in order to remove low quality reads $(\mathrm{Q}>30)$. The reads that passed the quality check were assembled against the Bos taurus genome (Ensembl Btau_4.0) using TopHat2 [11].

\section{Differential expression and pathway analysis}

Cuffdiff2 within the Cufflink package (v2.2.1) [12] was used to identify differentially expressed (DE) genes and genes with $\mathrm{a}+/-2$-fold change or more and with $\mathrm{p} \leq 0.05$ 
were considered significant and used in the further analysis. Ingenuity Pathway Analysis (IPA) (http://www.ingenuity. com/products/ipa) was applied for the functional annotation and pathway analysis.

\section{Realtime PCR verification}

150 ng RNA were converted into cDNA using superscript (Life Technologies) with $150 \mathrm{ng}$ oligo(dT) (Promega) according to the manufacturer's instructions. Fast SYBR Green Master mix (Life Technologies) was run according to the manufacturer's protocol, primers are available on request. GAPDH was used as a housekeeping gene and the expression fold-changes was calculated using the $2^{-\Delta \Delta C t}$ method.

\section{Abbreviations}

SBV: Schmallenberg virus; LAVC: La Crosse virus; AKBV: Akabane virus; CCV: Cache Valley virus; BUNV: Bunyamwera.

\section{Competing interests}

The authors declare that they have no competing interests.

\section{Authors' contributions}

ALB and JKS carried out the experimental work, apart from the library preparation and MiSeq sequencing that was performed by GW and MB. GB isolated and grew the bovine primary cells and RME contributed with the viruses used in this study. The bioinformatic analysis (quality, assambly, DE analysis) was perfomed by QG, the IPA analysis was carried out by QG and ALB. ES and MMF were involved in experimental discussions. ALB and AK concieved the study, designed the experiments and wrote the initial manuscrip draft. QG, GB, GW, JKS, MB, MMF, ES, RME and MP contributed to the draft and revising of the final manuscript. All authors read and approved the final manuscript.

\section{Acknowledgements}

This work was supported by the Swedish Research Council (Grant no. 2012-6555) (A-LB) and the UK Medical Research Council (AK).

\section{Author details}

${ }^{1}$ Section of Virology, Department of Biomedical Sciences and Veterinary Public Health, Swedish University of Agricultural Sciences, 75007 Uppsala, Sweden. ${ }^{2}$ MRC-University of Glasgow Centre for Virus Research, Glasgow G61 $1 \mathrm{QH}$, Scotland, UK. ${ }^{3}$ UCD Veterinary Science Centre, School of Veterinary Medicine, University College Dublin, Belfield, Dublin, Ireland.

Received: 18 February 2015 Accepted: 14 April 2015

Published online: 19 April 2015

\section{References}

1. Hoffmann B, Scheuch M, Hoper D, Jungblut R, Holsteg M, Schirrmeier H, et al. Novel orthobunyavirus in Cattle, Europe, 2011. Emerg Infect Dis. 2012;18(3):469-72.

2. Authority EFS. "Schmallenberg virus": analysis of the epidemiological data (November 2012). In: Supporting Publications, vol. EN-360. 2012. p. 22.

3. van den Brom R, Luttikholt SJ, Lievaart-Peterson K, Peperkamp NH, Mars MH, van der Poel $\mathrm{WH}$, et al. Epizootic of ovine congenital malformations associated with Schmallenberg virus infection. Tijdschr Diergeneeskd. 2012;137(2):106-11.

4. Elliott RM. Orthobunyaviruses: recent genetic and structural insights. Nat Rev Microbiol. 2014;12(10):673-85.

5. Blakqori G, Delhaye S, Habjan M, Blair CD, Sanchez-Vargas I, Olson KE, et al. La Crosse bunyavirus nonstructural protein NSs serves to suppress the type I interferon system of mammalian hosts. J Virol. 2007;81(10):4991-9.

6. Bridgen A, Weber F, Fazakerley JK, Elliott RM. Bunyamwera bunyavirus nonstructural protein NSs is a nonessential gene product that contributes to viral pathogenesis. Proc Natl Acad Sci U S A. 2001;98(2):664-9.
7. Ogawa Y, Sugiura K, Kato K, Tohya Y, Akashi H. Rescue of Akabane virus (family Bunyaviridae) entirely from cloned cDNAs by using RNA polymerase I. J Gen Virol. 2007;88(Pt 12):3385-90.

8. Elliott RM, Blakqori G, van Knippenberg IC, Koudriakova E, Li P, McLees A et al. Establishment of a reverse genetics system for Schmallenberg virus, a newly emerged orthobunyavirus in Europe. J Gen Virol. 2013;94(Pt 4):851-9.

9. Varela M, Schnettler E, Caporale M, Murgia C, Barry G, McFarlane M, et al. Schmallenberg virus pathogenesis, tropism and interaction with the innate immune system of the host. PLoS Pathog. 2013;9(1), e1003133.

10. Barry G, Varela M, Ratinier M, Blomstrom AL, Caporale M, Seehusen F, et al. NSs protein of Schmallenberg virus counteracts the antiviral response of the cell by inhibiting its transcriptional machinery. J Gen Virol. 2014;95(Pt 8):1640-6.

11. Kim D, Pertea G, Trapnell C, Pimentel H, Kelley R, Salzberg SL. TopHat2 accurate alignment of transcriptomes in the presence of insertions, deletions and gene fusions. Genome Biol. 2013;14(4):R36.

12. Trapnell $C$, Hendrickson DG, Sauvageau M, Goff L, Rinn JL, Pachter L. Differential analysis of gene regulation at transcript resolution with RNA-seq. Nat Biotechnol. 2013;31(1):46-53.

13. Seo JY, Yaneva R, Cresswell P. Viperin: a multifunctional, interferon-inducible protein that regulates virus replication. Cell Host Microbe. 2011;10(6):534-9.

14. Helbig KJ, Carr JM, Calvert JK, Wati S, Clarke JN, Eyre NS, et al. Viperin is induced following dengue virus type-2 (DENV-2) infection and has anti-viral actions requiring the C-terminal end of viperin. PLoS Negl Trop Dis. 2013;7(4), e2178.

15. Teng TS, Foo SS, Simamarta D, Lum FM, Teo TH, Lulla A, et al. Viperin restricts chikungunya virus replication and pathology. J Clin Invest. 2012;122(12):4447-60.

16. Wang $X$, Hinson ER, Cresswell $P$. The interferon-inducible protein viperin inhibits influenza virus release by perturbing lipid rafts. Cell Host Microbe. 2007;2(2):96-105

17. Helbig K, Beard MR. The role of viperin in the innate antiviral response. J Mol Biol. 2014:426(6):1210-9.

18. Zapata JC, Carrion Jr R, Patterson JL, Crasta O, Zhang Y, Mani S, et al. Transcriptome analysis of human peripheral blood mononuclear cells exposed to Lassa virus and to the attenuated Mopeia/Lassa reassortant 29 (ML29), a vaccine candidate. PLoS Negl Trop Dis. 2013;7(9), e2406.

19. Carlton-Smith C, Elliott RM. Viperin, MTAP44, and protein kinase $R$ contribute to the interferon-induced inhibition of Bunyamwera Orthobunyavirus replication. J Virol. 2012;86(21):11548-57.

20. Haller O, Staeheli P, Kochs G. Interferon-induced Mx proteins in antiviral host defense. Biochimie. 2007;89(6-7):812-8.

21. Rodrigues Hoffmann A, Dorniak P, Filant J, Dunlap KA, Bazer FW, de la Concha-Bermejillo A, et al. Ovine fetal immune response to Cache Valley virus infection. J Virol. 2013;87(10):5586-92.

22. Kochs $\mathrm{G}$, Janzen $\mathrm{C}$, Hohenberg $\mathrm{H}$, Haller O. Antivirally active MxA protein sequesters La Crosse virus nucleocapsid protein into perinuclear complexes. Proc Natl Acad Sci U S A. 2002;99(5):3153-8.

23. Sadler AJ, Williams BR. Interferon-inducible antiviral effectors. Nat Rev Immunol. 2008:8(7):559-68.

24. Fink J, Gu F, Ling L, Tolfvenstam T, Olfat F, Chin KC, et al. Host gene expression profiling of dengue virus infection in cell lines and patients. PLoS Negl Trop Dis. 2007;1(2), e86.

25. Sessions OM, Tan Y, Goh KC, Liu Y, Tan P, Rozen S, et al. Host cell transcriptome profile during wild-type and attenuated dengue virus infection. PLoS Negl Trop Dis. 2013;7(3), e2107.

26. Kraut N, Snider L, Chen CM, Tapscott SJ, Groudine M. Requirement of the mouse I-mfa gene for placental development and skeletal patterning. EMBO J. 1998;17(21):6276-88.

27. Gillespie Jl, Greenwell JR, Johnson C. Agonist-induced fluctuations in cytoplasmic calcium in primary cultures of bovine endothelial cells. Exp Physiol. 1991;76(5):667-76. 